\title{
Dark and bright modes manipulation for plasmon-triggered photonic devices
}

\author{
S. Panaro*,a, A. Nazir ${ }^{\mathrm{a}}$, C. Liberale ${ }^{\mathrm{a}}$, H. Wang ${ }^{\mathrm{a}, \mathrm{b}}$, F. De Angelis ${ }^{\mathrm{a}}$, R. Proietti Zaccaria ${ }^{\mathrm{a}}$, E. Di \\ Fabrizio $^{\text {c,d }}$, A. Toma ${ }^{a}$ \\ ${ }^{\mathrm{a}}$ Istituto Italiano di Tecnologia, via Morego 30, I-16163 Genova, Italy; ${ }^{\mathrm{b}}$ College of Electronic \\ Science and Engineering, Jilin University, 2699 Qianjin Street, Changchun 130012, China; ${ }^{\mathrm{P}} \mathrm{PSE}$ and \\ BESE Divisions, KAUST, King Abdullah University of Science and Technology, Thuwall, $23955-$

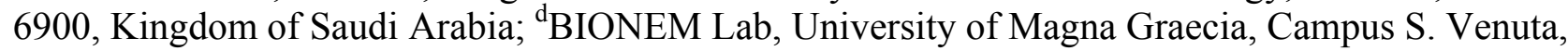 \\ Germaneto, viale Europa, I-88100 Catanzaro, Italy \\ *email address: simone.panaro@,iit.it
}

\begin{abstract}
In the last decade, several efforts have been spent in the study of near-field coupled systems, in order to induce hybridization of plasmonic modes. Within this context, particular attention has been recently paid on the possibility to couple conventional bright and dark modes. As a result of such phenomenon, a Fano resonance appears as a characteristic sharp dip in the scattering spectra. Here we show how, gradually coupling a single rod-like nanostructure to an aligned nanoantenna dimer, it is possible to induce the near-field activation of an anti-bonding dark mode. The high polarization sensitivity presented by the far-field response of T-shape trimer, combined with the sharp Fano resonance sustained by this plasmonic device, opens interesting perspectives towards a new era of photonic devices.
\end{abstract}

Keywords: plasmonics; localized surface plasmon; Fano resonance.

\section{INTRODUCTION}

The polarization dependency of localized surface plasmons (LSPs) is a common property presented by rod-like nanoantennas, which can be ascribed to the associated resonator length of a Fabry-Perot cavity ${ }^{1-5}$. The possibility to excite LSPs in different spectral windows by rotating the light polarization has been recently employed for the optimization of multiband sensing devices ${ }^{6,7}$. However conventional plasmonic systems are generally characterized by a broad electromagnetic (EM) response which, apart from cases of high aspect ratio geometries ${ }^{1,6,8,9}$, tends to reduce the optical spectral selectivity associated to anisotropic nanostructures.

A possible strategy which can be adopted in order to obtain spectral responses with sharper line-shape consists in inducing plasmonic hybridization of bright and dark (non-radiating) modes ${ }^{10-13}$. In fact dark modes, due to their weak coupling to free radiation, present narrow band spectral responses ${ }^{14-18}$. One of the simplest plasmonic systems which can sustain dark oscillations is the aligned nanoantenna dimer ${ }^{19}$. This elemental nanoassembly presents two low order hybridized modes: (i) the low energy (bonding) mode is characterized by the in-phase oscillation of the localized surface plasmons (LSPs) associated to each nanoantenna arm, and (ii) the high energy (anti-bonding) mode is determined by the mutually out-of-phase oscillation of LSPs.

In this work, we theoretically show how it is possible to induce the hybridization of a single nanoantenna bright mode and a dimer dark mode, by gradually putting the single nanoantenna in strong near-field coupling to the dimer nanoassembly, obtaining a T-shape trimer. After having shown the high spectral selectivity of the T-shape device, by means of a polarization analysis we show the strong anisotropy of the trimer extinction efficiency. Finally we observe how the scattering efficiency of the system tends to zero in a spectral region between the two trimer scattering peaks.

Plasmonics: Metallic Nanostructures and Their Optical Properties XII, edited by Allan D. Boardman, Proc. of SPIE Vol. 9163, 91633M - @ 2014 SPIE CCC code: $0277-786 \mathrm{X} / 14 / \$ 18 \cdot$ doi: $10.1117 / 12.2061187$ 


\section{T-SHAPE TRIMER: FROM WEAK TO STRONG COUPLING REGIME}

We considered a system composed of a single gold nanoantenna with length $150 \mathrm{~nm}$, width and height $60 \mathrm{~nm}$. Similarly, a co-planar aligned nanoantenna dimer (each antenna presents the same geometrical parameters of the single one) is defined with main axis perpendicular to the single antenna long axis (see the cartoon in Figure 1(a)). The distance between the single antenna and the center of the dimer gap is defined $\zeta$. We performed electromagnetic (EM) simulation in frequency domain on the trimer system by means of a commercial simulative software (CST Studio Suite 2012). Initially we set the linear polarization of incident radiation (normally incident to the plane of the system) parallel to the single nanoantenna long axis (see Figure 1(a)) and we studied both the near- and the far-field response of the system as a function of the coupling parameter $\zeta$.

Observing the extinction efficiency spectrum of the trimer for $\zeta=100 \mathrm{~nm}$, we can notice a single peak centered around $800 \mathrm{~nm}$ (black curve in Figure 1(b)). From the near-field distribution associated to the system in resonance condition, we can see how the single nanoantenna presents two identical electric hot-spots at its apexes (Fig. 1(c)). The nanoantenna dimer is in extremely weak EM interaction with the single antenna. By gradually reducing the $\zeta$ parameter, a bimodal behavior in the extinction spectrum clearly appears around $\zeta=70 \mathrm{~nm}$ (blue curve in Figure 1(b)). At last, for $\zeta$ $=15 \mathrm{~nm}$ (inter-particle gaps of around $10 \mathrm{~nm}$ ), we obtain a strongly coupled T-shape nanoantenna trimer and we can appreciate an associated extinction spectrum presenting two distinct peaks respectively at $700 \mathrm{~nm}$ and $900 \mathrm{~nm}$ (see lower purple curve in Figure 1(b)). By simulating the near-field response of the system for incoming EM radiation at $\lambda=900$ nm (bonding resonance condition), we can clearly see two intense hot-spots inside the inter-particle gaps (Fig. 1(d)), which is the typical response of a strongly coupled nano-assembly. Around the anti-bonding peak $(700 \mathrm{~nm})$ instead, the near-field response of the T-shape trimer is different and shows a strong plasmonic activity inside the vertical antenna dimer. In this condition, we can observe how the LSPs associated to the aligned dimer sub-system are exclusively triggered by near-field channel. For symmetry reasons, the mode sustained by the aligned dimer is dark ${ }^{19}$.
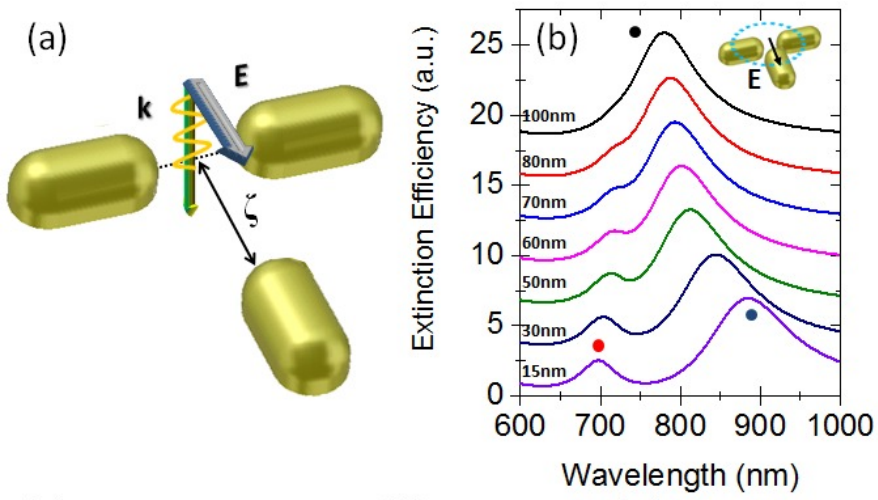

(c)

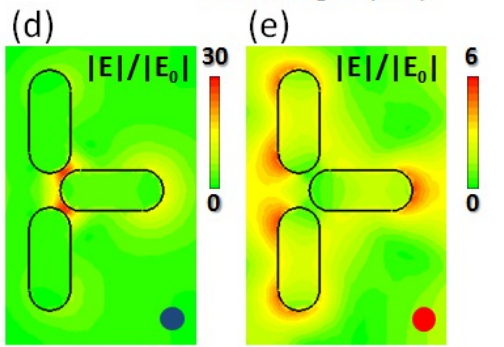

Figure 1. (a) Sketch of the T-shape trimer (the long axis of the single antenna is passing thorugh the center of the dimer gap), together with the optical configuration of incident light. (b) Extinction efficiency spectra of T-shape trimer as a function of $\zeta$ parameter. (c) 2-D plot of electric field enhancement distribution in resonant condition of a weakly coupled T-shape trimer $(\zeta=100 \mathrm{~nm})$, simulated on a plane parallel to the substrate and passing through the center of the system. (d,e) 2-D plots of electric field enhancement distribution, respectively in bonding and anti-bonding resonant condition, of a strongly coupled T-shape trimer $(\zeta=15$ nm), simulated on a plane parallel to the substrate and passing through the center of the system.

\section{POLARIZATION DEPENDENCY AND SCATTERING EFFICIENCY}

T-shape trimers in strong coupled configuration represent a nano-system endowed with peculiar and intriguing optical properties. The plamonic modes which determine the spectral response presented in Figure 1(b) are critically dependent on the polarization states of the incoming light. In order to highlight this aspect, we performed far-field simulations on a 
single assembly, varying the polarization in the range of $-45^{\circ}<\theta<45^{\circ}$ at steps of $5^{\circ}$ (see sketch in Figure 2(a)), and we plotted the corresponding extinction efficiency spectra (Fig. 2(b)).

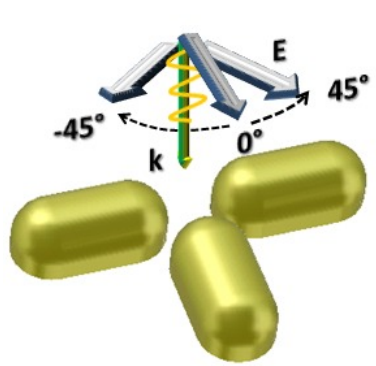

(a)

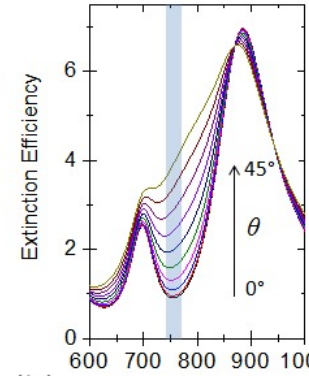

(b)

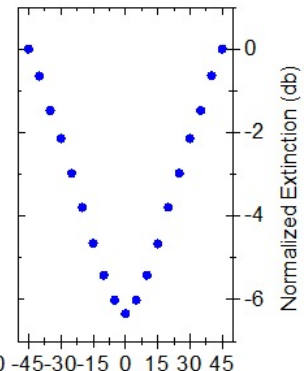

(c) $\theta$ (deg)

Figure 2. (a) Sketch of the T-shape trimer in strong coupling condition, indicating the sweep of incident light polarization. (b) Extinction efficiency spectra of T-shape trimer as a function of incident light polarization. (c) Plot reporting the normalized extinction efficiency (ratio between the actual value and the maximum value collected for $\theta= \pm 45^{\circ}$ ) values calculated around the local minimum of the spectra (see vertical azure band in Figure 2(b)), as a function of the polarization angle $\theta$.

We showed spectra only for the positive angle sweep (Fig. 2(b)), since the system is symmetric for inversion of the angle sign. When $\theta=0^{\circ}$, a sharp extinction dip is observed around $750 \mathrm{~nm}$. By gradually increasing $\theta$, the extinction efficiency inside the dip rises becoming comparable with the bonding and anti-bonding resonance peaks. In $\theta=45^{\circ}$ condition we are exciting the bright modes sustained by the L-shape dimer sub-elements ${ }^{19,20}$ and the aligned nanoantenna dark mode is completely suppressed. In this analysis, the high polarization sensistivity and spectral selectivity of T-shape trimer clearly emerges.

In order to evaluate the optical switching capability of T-shape nanoantenna trimer, we focused around the local extinction minimum (see azure vertical band in Figure 2(b)) and plotted the extinction efficiency value as a function of the polarization angle, for $-45^{\circ}<\theta<45^{\circ}$ (Fig. 2(c)). The values have been normalized to the maximum extinction collected and have been expressed in decibel. The plot obtained shows a variation of more than $6 \mathrm{db}$ from the "extinction mode" $\left(\theta= \pm 45^{\circ}\right)$ to the "transmission mode" $\left(\theta=0^{\circ}\right)$.

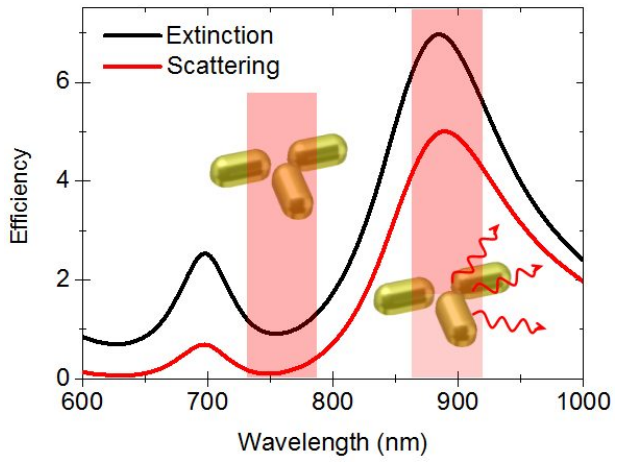

Figure 3. Extinction and scattering efficiency spectra of T-shape trimer nano-assembly in $\theta=0^{\circ}$ polarization condition.

The rotation of the light polarization induces a significant change in the optical response of the T-shape trimer. This phenomenon is different from rod-like nanoantenna case, where the polarization rotation induces a LSPR shift. In this case, the entire extinction spectrum of the trimer does not shift but an extra feature seems to appear in the line-shape during the polarization sweep from the $\pm 45^{\circ}$ to the $0^{\circ}$ condition. Systems like this have been recently studied with the name of plasmonic Fano resonant nanostructures ${ }^{21,22}$. They present an extra-transparency originating from the hybridization of bright and dark modes, with direct impact on their own scattering properties ${ }^{23}$.

In order to have a deeper insight on the radiative properties of T-shape trimer, we simulated the scattering efficiency spectrum of the system for $\theta=0^{\circ}$, and plotted it in Figure 3 (red curve). The corresponding extinction efficiency spectrum has been reported in the same graph (black curve). From both the spectra we can appreciate the two peaks ascribed to the bonding $(900 \mathrm{~nm})$ and anti-bonding $(700 \mathrm{~nm})$ trimer modes, whose near-field distributions have been shown in Figures 1(d,e). Moreover, focusing on the local minimum around $750 \mathrm{~nm}$, we can appreciate how the scattering efficiency of the device tends to zero. Apart from internal damping contributions (absorption efficiency), the optical 
response of the trimer system around $750 \mathrm{~nm}$ can be considered as an electromagnetically induced transparency ${ }^{24}$ resulting from the hybridization of the single antenna bright mode and the aligned dimer dark mode.

\section{CONCLUSION}

In conclusion, we have theoretically studied a T-shape plasmonic nano-assembly composed by three indentical rod-like nanoantennas put in strong coupling condition. Firstly we have observed the change in the far-field response of the trimer by gradually increasing the near-field coupling between the bright and dark modes. Secondly we have analyzed the effect of polarization on the EM response of the system, highlighting the high spectral selectivity of the device and its polarization sensistivity. At last we have focused our attention on the scattering efficiency spectrum of T-shape trimer noticing how T-shape trimer presents electromagnetically induced transparency in the visible/near-infrared region. The sharpness of the Fano resonance and the feasible control of its spectral position candidate T-shape nanoantennas as elemental unit for conceiving spectral selective photonic devices with potential applicability in ultrafast signal multiplexing.

\section{REFERENCES}

[1] De Angelis, F., Malerba, M., Patrini, M., Miele, E., Das, G., Toma, A., Proietti Zaccaria, R. and Di Fabrizio, E., "3D Hollow Nanostructures as Building Blocks for Multifunctional Plasmonics", Nano Lett. 13(8), 3553-3558 (2013).

[2] Neubrech, F., Kolb, T., Lovrincic, R., Fahsold, G., Pucci, A., Aizpurua, J., Cornelius, T. W., Toimil-Molares, M. E., Neumann, R. and Karim, S., "Resonances of individual metal nanowires in the infrared", Appl. Phys. Lett. 89(25), 253104 (2006).

[3] Bryant, G. W., Garcia de Abajo, F. J. and Aizpurua, J., "Mapping the Plasmon Resonances of Metallic Nanoantennas", Nano Lett. 8(2), 631-636 (2008).

[4] Biagioni, P., Huang, J. S. and Hecht, B., "Nanoantennas for visible and infrared radiation", Rep. Prog. Phys. 75(2), 024402 (2012).

[5] Alonso-Gonzalez, P., Albella, P., Neubrech, F., Huck, C., Chen, J., Golmar, F., Casanova, F., Hueso, L. E., Pucci, A., Aizpurua, J. and Hillenbrand, R., "Experimental verification of the spectral shift between near- and far-field peak intensities of plasmonic infrared nanoantennas", Phys. Rev. Lett. 110(20), 203902 (2013).

[6] D’Andrea, C., Bochterle, J., Toma, A., Huck, C., Neubrech, F., Messina, E., Fazio, B., Maragò, O. M., Di Fabrizio, E., Lamy de La Chapelle, M., Gucciardi, P. G. and Pucci, A., "Optical Nanoantennas for Multiband Surface-Enhanced Infrared and Raman Spectroscopy", ACS Nano 7(4), 3522-3531 (2013).

[7] Gopalakrishnan, A., Malerba, M., Tuccio, S., Panaro, S., Miele, E., Chirumamilla, M., Santoriello, S., Dorigoni, C., Giugni, A., Proietti Zaccaria, R., Liberale, C., De Angelis, F., Razzari, L., Khrane, R., Toma, A., Das, G. and Di Fabrizio, E., "Nanoplasmonic structures for biophotonic applications: SERS overview", Ann. Phys. 524(11), 620-636 (2012).

[8] Proietti Zaccaria, R., De Angelis, F., Toma, A., Razzari, L., Alabastri, A., Das, G., Liberale, C. and Di Fabrizio, E., "Surface plasmon polariton compression through radially and linearly polarized source", Opt. Lett. 37(4), 545-547 (2012).

[9] Giugni, A., Torre, B., Toma, A., Francardi M., Malerba, M., Alabastri, A., Proietti Zaccaria, R., Stockman, M. I. and Di Fabrizio, E., "Hot-electron nanoscopy using adiabatic compression of surface plasmons", Nat. Nanotechnol. 8(11), 845-852 (2013).

[10] Chirumamilla, M., Toma, A., Gopalakrishnan, A., Das, G., Proietti Zaccaria, R., Krahne, R., Rondanina, E., Leoncini, M., Liberale, C., De Angelis, F. and Di Fabrizio, E., "3D Nanostar Dimers with a Sub-10-nm Gap for Single-/Few-Molecule Surface-Enhanced Raman Scattering”, Adv. Mater. 26(15), 2353-2358 (2014).

[11] Chang, W.-S., Lassiter, J. B., Swanglap, P., Sobhani, H., Khatua, S., Nordlander, P., Halas, N. J. and Link, S., "A plasmonic Fano switch", Nano Lett. 12(9), 4977-4982 (2012).

[12] Lassiter, J. B., Sobhani, H., Knight, M. W., Mielczarek, W. S., Nordlander, P. and Halas, N. J., "Designing and deconstructing the Fano lineshape in plasmonic nanoclusters", Nano Lett. 12(2), 1058-1062 (2012).

[13] Habteyes, T. G., Dhuey, S., Cabrini, S., Schuck, P. J., Leone, S. R., "Theta-Shaped Plasmonic Nanostructures: Bringing "Dark" Multipole Plasmon Resonances into Action via Conductive Coupling", Nano Lett. 11(4), 1819-1825 (2011). 
[14] Neubrech, F., Weber, D., Katzmann, J., Huck, C., Toma, A., Di Fabrizio, E., Pucci, A. and Hartling, T., "Infrared optical properties of nanoantenna dimers with photochemically narrowed gaps in the $5 \mathrm{~nm}$ regime", ACS Nano 6(8), 7326-7332 (2012).

[15] Hao, F., Sonnefraud, Y., Van Dorpe, P., Maier, S. A., Halas, N. J. and Nordlander, P., "Symmetry Breaking in Plasmonic Nanocavities: Subradiant LSPR Sensing and Tunable Fano Resonance", Nano Lett. 8(11), 39833988 (2008).

[16] Ye, J., Wen, F., Sobhani, H., Lassiter, J. B., Van Dorpe, P., Nordlander, P. and Halas, N. J., "Plasmonic Nanoclusters: Near Field Properties of the Fano Resonance Interrogated with SERS", Nano Lett. 12(3), 16601667 (2012).

[17] Neubrech, F., Garcia-Etxarri, A., Weber, D., Bochterle, J., Shen, H., Lamy de la Chapelle, M., Bryant, G. W., Aizpurua, J. and Pucci, A., "Defect-induced activation of symmetry forbidden infrared resonances in individual metallic nanorods", Appl. Phys. Lett. 96(21), 213111 (2010).

[18] Schmidt, M. K., Mackowski, S. and Aizpurua, J., "Control of single emitter radiation by polarization- and position-dependent activation of dark antenna modes", Opt. Lett. 37(6), 1017-1019 (2012).

[19] Panaro, S., Nazir, A., Liberale, C., Das, G., Wang, H., De Angelis, F., Proietti Zaccaria, R., Di Fabrizio, E. and Toma, A., "Dark to Bright Mode Conversion on Dipolar Nanoantennas: A Symmetry-Breaking Approach", ACS Photonics 1(4), 310-314 (2014).

[20] Panaro, S., Toma, A., Proietti Zaccaria R., Chirumamilla, M., Saeed, A., Razzari, L., Das, G., Liberale, C., De Angelis, F. and Di Fabrizio, E., "Design and top-down fabrication of metallic L-shape gap nanoantennas supporting plasmon-polariton modes", Microelectron. Eng. 111, 91-95 (2013).

[21] Nazir, A., Panaro, S., Proietti Zaccaria, R., Liberale, C., De Angelis, F. and Toma, A., "Fano Coil-Type Resonance for Magnetic Hot-Spot Generation", Nano Lett. 14(6), 3166-3171 (2014).

[22] Lassiter, J. B., Sobhani, H., Knight, M. W., Mielczarek, W. S., Nordlander, P. and Halas, N. J., "Designing and deconstructing the Fano lineshape in plasmonic nanoclusters", Nano Lett. 12(2), 1058-1062 (2012).

[23] Luk'yanchuk, B., Zheludev, N. I., Maier, S. A., Halas, N. J., Nordlander, P., Giessen, H. and Chong, C. T., "The Fano resonance in plasmonic nanostructures and metamaterials", Nat. Mater. 9(9), 707-715 (2010).

[24]Zhang, S., Genov, D. A., Wang, Y., Liu, M. and Zhang, X., "Plasmon-induced transparency in metamaterials", Phys. Rev. Lett., 101(4), 047401 (2008). 